\title{
Error Growth Patterns in Systems with Spatial Chaos: From Coupled Map Lattices to Global Weather Models
}

\author{
C. Primo, ${ }^{1,2}$ I. G. Szendro, ${ }^{2}$ M. A. Rodríguez, ${ }^{2}$ and J. M. Gutiérrez ${ }^{3}$ \\ ${ }^{1}$ Department of Meteorology, University of Reading, Earley Gate, Reading RG6 6BB, United Kingdom \\ ${ }^{2}$ Instituto de Física de Cantabria, CSIC-UNICAN, Santander E-39005, Spain \\ ${ }^{3}$ Department of Applied Mathematics. University of Cantabria, 39005, Santander, Spain
}

(Received 30 August 2006; published 6 March 2007)

Error growth in spatiotemporal chaotic systems is investigated by analyzing the interplay between temporal and spatial dynamics. The spatial correlation and localization of relative fluctuations grow and decay indicating two different regimes, before and after saturation by nonlinear effects. This general behavior is shown to hold both in simple coupled map lattices and in global weather models. This explains the increasing or decreasing trends previously observed in the exponential growth rate of these spatiotemporal systems.

PACS numbers: 92.60.Wc, 05.45.Pq, 68.35.Ct

During the last two decades, ensemble prediction systems (EPS) have become the main tool for dealing with uncertainty in weather forecasts [1]. Under this framework, a probabilistic forecast is given by an ensemble of simulations computed by sampling the different sources of uncertainty. For instance, different techniques such as bred vectors and singular vectors have been proposed to efficiently sample the uncertainty related to the initial condition assessment by introducing appropriate perturbations. Although much work has been devoted to the generation of such ensembles, still little is known about the spatiotemporal evolution of perturbations, which grow exponentially in time and form characteristic spatial patterns. The characterization of these patterns is necessary for improving the predictability in spatial chaotic systems.

Several "toy models" such as coupled map lattices [2] or the family of Lorenz models [3] have been extensively used in the literature to explore the basic features of spatiotemporal dynamics, including the evolution of perturbations due to errors in the initial conditions. The global characterization of these perturbations is defined by means of the distance between two typical trajectories (as, for instance, the control and a perturbed member in perfectmodel EPS approaches) which is a spatial pattern fluctuating in time. Properties of spatiotemporal dynamics can be extracted from these simple "toy models" and extrapolated to more complex models (such as weather models). A recent Letter [4] analyzes the exponential growth rate of perturbations for different spatiotemporal systems, from operational weather models to simplified quasigeostrophic and simple Lorenz models. They report a different behavior between weather models, exhibiting convex (decreasing) growth-rate curves, and simpler models, exhibiting flat or concave (increasing) curves. Such a difference is interpreted in terms of the multiple scales typical of weather models and the presence of multiple types of instabilities that dominate at different amplitude ranges. However, it is clear that a more complete picture with some theoretical support is needed to uncover the relevant factors for this concave-convex character.

In this Letter we give an explanation of this problem in terms of the interplay between temporal and spatial evolution of perturbations. We show that the same model can exhibit either increasing or decreasing growth rates depending on the choice of the initial perturbation.

We argue that the spatiotemporal evolution of perturbations in space and time can be explained as a transient of the relative fluctuations obtained by removing the global growth. In a first stage, infinitesimal fluctuations evolve in the linear tangent space and tend to a correlated and localized pattern defined by a set of linear evolution equations driven by multiplicative varying factors. In a second stage, nonlinear saturation effects become relevant, and correlation and localization progressively decay. These two regimes characterize the spatiotemporal evolution of fluctuations for different choices of the initial perturbations. In particular, we show that the increasing or decreasing curve of the growth rate is an effect of the initial localization in 1D coupled map lattices, or the anisotropy in weather models.

We consider a general spatiotemporal system of coupled space-time fields $\left\{\Phi_{i}(s, t)\right\}$, where $s$ represents the location in space and $t$ in time. This system evolves in time according to $\dot{\Phi}_{i}(s, t)=F_{i}\left(\left\{\Phi_{j}(s, t)\right\}\right), F_{i}$ being generic functionals of the fields. The evolution of fluctuations or perturbations, $\left\{\delta \Phi_{i}(s, 0)\right\}$, due to errors in the initial conditions, follows a multiplicative linear equation in its infinitesimal initial phase given by $\delta \dot{\Phi}_{i}(s, t)=$ $\int \frac{\delta F_{i}(s, t)}{\delta \Phi_{j}\left(s^{\prime}, t^{\prime}\right)} \delta \Phi_{j}\left(s^{\prime}, t^{\prime}\right) d s^{\prime} d t^{\prime}$. If local functionals including dissipative and convective effects are assumed to be essential ingredients, then the infinitesimal evolution of fluctuations follows the equation

$$
\delta \dot{\Phi}_{i}(s, t)=\sum_{j}\left(\nabla D_{i j} \nabla \delta \Phi_{j}+V_{i j} \nabla \delta \Phi_{j}+K_{i j} \delta \Phi_{j}\right)
$$

where the coefficients $D, V, K$ are functionals of the 
unperturbed fields $\Phi_{i}(s, t)$. It has been shown that in the case of chaotic evolution the fluctuations grow exponentially, and such coefficients behave as stochastic fields [5].

Fluctuations are usually analyzed in terms of the temporal component. However, as we show below, spatial dynamics (growth and decay of correlations) also play an important role in spatiotemporal systems. To analyze this component we consider $\delta \Phi_{i}(s, t)=E_{i}(t) \delta u_{i}(s, t)$. On the one hand, $E_{i}(t)$ is the temporal amplitude of the fluctuation, which we define as the geometric mean $E_{i}(t)=$ $\left(\prod_{s}\left|\delta \Phi_{i}(s, t)\right|\right)^{1 / L}$, where $L$ is the system size, and grows asymptotically as $E_{i}(t) \sim a_{i} \exp \lambda t$ defining the spatially averaged separation between two trajectories of the field $\Phi_{i}(s, t)$, i.e., the global error growth [6]. On the other hand, the reduced fluctuations $\delta u_{i}(s, t)$ retain the information of the relative growth in space and according to (1) evolve as:

$$
\dot{\delta} u_{i}=\frac{E_{j}}{E_{i}}\left[\nabla D_{i j} \nabla \delta u_{j}+V_{i j} \nabla \delta u_{j}+\left(K_{i j}-\frac{\dot{E}_{i}}{E_{i}} \delta_{i j}\right) \delta u_{j}\right],
$$

where $\delta_{i j}$ is the Kronecker delta. [Note that from (2)-(4) summation over index $j$ is still assumed.] In the asymptotic limit the relative amplitudes $E_{j} / E_{i}$ and the exponential growth rate $\dot{E}_{i} / E_{i}$ tend to the constant values $a_{j} / a_{i}$ and $\lambda$, respectively. However, the reduced fluctuations $\delta u_{i}$ tend to a fluctuating stationary pattern which is reached sooner or later depending not only on the amplitude, but also on the spatial form of the initial perturbation. This spatial component plays a central role in the explanation of the growth of perturbations and it can be analyzed within the framework of rough interfaces [8].

To this aim, we consider the Hopf-Cole transformation of (2) given by the functions $h_{i}(s, t)=\ln \left|\delta u_{i}(s, t)\right|$ :

$$
\begin{aligned}
\dot{h}_{i}= & \lambda_{i j}\left[\nabla D_{i j} \nabla h_{j}+D_{i j}\left(\nabla h_{j}\right)^{2}+V_{i j} \nabla h_{j}\right. \\
& \left.+\left(K_{i j}-\frac{\dot{E}_{i}}{E_{i}} \delta_{i j}\right)\right]
\end{aligned}
$$

where $\lambda_{i j}=\delta \phi_{j} / \delta \phi_{i}$. With this transformation we get an additive character for the fluctuation of $h_{i}(s, t)$. The diagonal part $\left[\dot{h}_{i}(s, t)\right]_{d}=\nabla D_{i i} \nabla h_{i}+D_{i i}\left(\nabla h_{i}\right)^{2}+V_{i i} \nabla h_{i}+$ $\left(K_{i i}-\dot{E}_{i} / E_{i}\right)$ represents the growth of interfaces in the Kardar-Parisi-Zhang (KPZ) universality, whereas the nondiagonal part $\left[\dot{h}_{i}(s, t)\right]_{\mathrm{nd}}=\lambda_{i j}\left[\nabla D_{i j} \nabla h_{j}+D_{i j}\left(\nabla h_{j}\right)^{2}+\right.$ $\left.V_{i j} \nabla h_{j}+K_{i j}\right]$ accounts for cross-correlation effects between distinct fields that can lead to anisotropy and bounds in the growth.

The spatiotemporal evolution of statistics in rough interfaces follows precise power laws with exponents given by a reduced set of "universality" classes, such as Edwards-Wilkinson (EW) and KPZ (see [8] for more details). According to (3) we can expect anisotropic, bounded, simple universality classes such as EW or KPZ. This theoretical formulation allows the proper analysis of spatiotemporal characteristics, such as the error growth rate described in [4]. The exponential growth rate $\dot{E}_{i} / E_{i}$ can be easily obtained by spatially averaging both members of (3). (Note that $\bar{h}_{i}=0$, since $\prod_{s}\left|\delta u_{i}(s, t)\right|=1$, where overlines indicate spatial averages.) In a closed system in which $\overline{\lambda_{i j} \nabla D_{i j} \nabla h_{j}}=\overline{\lambda_{i j} V_{i j} \nabla h_{j}}=0$, the exponential growth rate is composed of two elements:

$$
\frac{\dot{E}_{i}}{E_{i}}=\overline{\lambda_{i j} D_{i j}\left(\nabla h_{j}\right)^{2}}+\overline{\lambda_{i j} K_{i j}}
$$

called the local exponential growth and the spatially averaged nonlinear KPZ term $\left(\nabla h_{j}\right)^{2}$. Note that, as assumed in [4], there is an evolution equation for the logarithmic growth rate but it depends explicitly on the spatial dynamics through the KPZ term. Both terms have important implications in the ensemble dynamics, as described in the following sections.

ID coupled map lattices. - We first consider the case of coupled chains of 1D chaotic maps. For these spatiotemporal systems Eq. (3) reduces to the diagonal part $(i=1)$, which belongs to the KPZ universality class. Thus, the stationary state is a fluctuating fractal curve (the saturated interface) with a rough exponent $\alpha=1 / 2$. Spatial patterns of this kind can be fully characterized by the power spectral density (PSD) defined as $S(k, t)=\langle h(k, t) h(-k, t)\rangle$, $h(k, t)$ being the Fourier transform of $h(x, t)$ and $\langle.$.$\rangle repre-$ senting the average over samples, as, for instance, ensembles. Rough curves with exponent $\alpha$ have a PSD given by $S(k) \sim \frac{S_{0}}{k^{2 \alpha+1}}$, where the constant $S_{0}$ is only dependent on the details of the system.

To illustrate these ideas we use a simple array of diffusively coupled logistic maps with three distinct initial conditions. Readers interested in details of the model as well as in the evolution of fluctuations are referred to [9]. The three initial conditions are chosen to show the different ways of approaching the stationary configuration of the relative fluctuation. In all cases we use initial ensembles of perturbations $\{\delta u(s, 0)\}$ with the same spatial amplitude. In case $A$ we use fully correlated perturbations obtained as bred vectors. In cases $B$ and $C$ we consider spatially uncorrelated random perturbations. Moreover, the statistical distribution of $A$ and $B$ is log-normal (localized) whereas $C$ is Gaussian.

The evolution of spectra for the three cases is shown in Fig. 1 (five different times are marked with different symbols). In case $A$, the spectrum remains unchanged since it is in the stationary condition from the beginning. In cases $B$ and $C$ the initial spectrum $S(k, 1)$ represented by $\bullet$ evolves to reach the stationary configuration $S(k)=\frac{S_{0}}{k^{2}}$ represented by $*$. This evolution occurs first for small scales, large values of $k$, and then for large scales. The initial spectra in cases $B$ and $C$ are obviously plane with a value that is, respectively, in the half and the bottom of the stationary spectral shape. The evolution in case $B$ occurs reducing the area of the spectrum whereas in $C$ such area becomes greater. According to the Percival identity, the area of the spectrum $\int S(k, t) d k$ coincides with the squared width of 

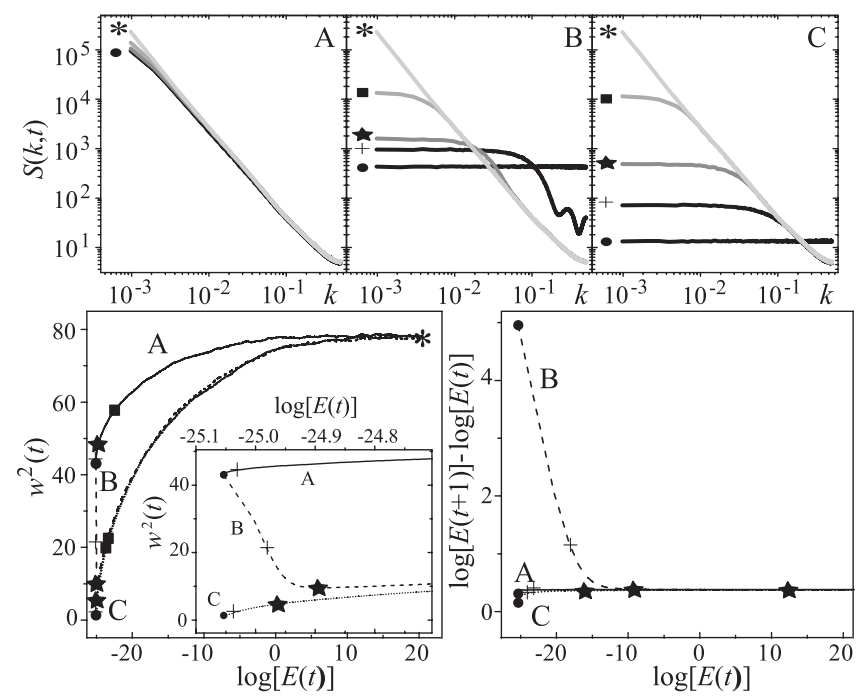

FIG. 1. Simulations of the linear infinitesimal fluctuations (2) for a chain of 1024 coupled logistic maps with three different initial perturbations, indicated by $A, B$, and $C$. Upper panels show the power spectra $S$ in $\log$-log plots for 5 times $\left(t_{\bullet}<t_{+}<\right.$ $t_{\star}<t_{\square}<t_{*}$ ). Lower panels show the plot of the squared width $w^{2}$ and the logarithmic growth rate $\dot{E} / E$ against the logarithmic amplitude $\log [E(t)]$. The five symbols indicate five different times in the evolution. The results are averages over 2000 samples. The inset in the lower panel is an amplification of the initial behavior corresponding to small amplitudes.

the interface $w^{2}(t)=\overline{[h(s, t)-\overline{h(s, t)}]^{2}}$ [8]. Similarly, the averaged KPZ term $\overline{(\nabla h)^{2}}$ is the second moment of the spectrum $\int k^{2} S(k, t) d k$. Hence the squared width $w^{2}$ and the logarithmic growth rate $\dot{E} / E \sim \overline{(\nabla h)^{2}}$ can be used to measure the strength of relative fluctuations in large and small spatial scales, respectively. Lower panels of Fig. 1 show both $w^{2}(t)$ and $\dot{E} / E$ against the logarithmic amplitude, which is asymptotically proportional to time, for the three initial conditions. Even with random initial conditions, both the width of the interface and the logarithmic growth rate exhibit increasing or decreasing curves depending on the initial distribution.

For the nonlinear case, the evolution of relative fluctuations in the linear phase is identical since it is independent of the initial amplitude $E(0)$. Nevertheless, after a time $t_{l}$ in which nonlinear terms become relevant, linearized equations like (2) are not valid. After this time, correlation and localization progressively decay due to nonlinear saturation effects [9]. Figure 2 shows the complete evolution of finite fluctuations. The two regimes of infinitesimal and finite fluctuations are easily identified by comparison with Fig. 1. In agreement with the explained evolution of the spectra, in the first regime of the evolution the lower panels show increasing, flat, or decreasing curves depending on the choice of the initial perturbation. In the second regime, which is dominated by saturation effects, the correlation $w^{2}(t)$ accumulated in the first regime progressively decays to the same value in all cases.
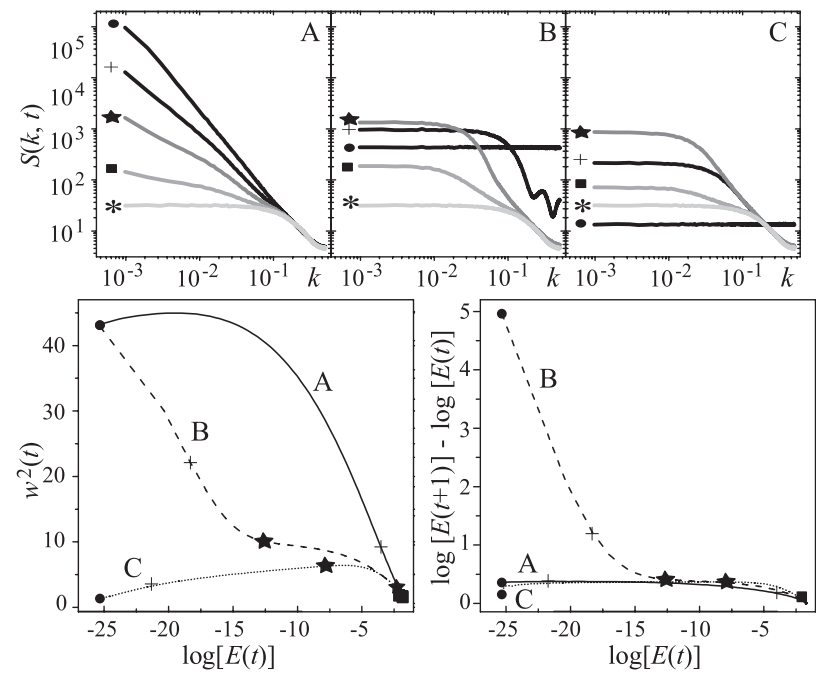

FIG. 2. As in Fig. 1 but for the original nonlinear equations.

It is worth remarking that in this framework both the spatial and temporal patterns have universal forms derived from dynamic scaling laws. For instance, in the standard case $C$ of initial Gaussian fluctuations, the spectra follow the scaling law $S(k, t)=\frac{S_{0}}{k^{2 \alpha+1}} f\left(k t^{1 / z}\right), f(u)$ being a universal scaling function. Integrating the spectra we get $w(t) \sim$ $t^{\alpha / z}, \quad \dot{E} / E \sim K+\overline{(\nabla h)^{2}} \sim K+t^{-2 \beta}$. These are wellknown scaling laws of the width and velocity of interfaces in the 1D KPZ universality [10].

Global weather models. - In the case of general highdimensional systems, Eq. (3) contains both diagonal and nondiagonal terms, resulting in anisotropic surfaces growing with coupled boundaries. However, they may exhibit a behavior similar to the previous case if there exists an exponential growth of initial perturbations. In this section we illustrate this fact by considering two operational EPS products of the European Centre for Medium-Range Forecast (ECMWF): the medium-range and the monthly forecast systems [11]. These systems provide predictions ten and 30 days ahead, respectively. In these two models perturbations are introduced in the atmospheric component using a particular perturbation scheme for the initial conditions (the singular vectors method). Thus, the initial perturbations possess a certain spatial correlation structure.

The main difference of these complex models with the 1D lattices considered in the previous sections is their anisotropy, which can be observed in any spatial representation. For instance, plots of the local width of the logarithmic fluctuations (member minus control) exhibit a strong lateral isotropy with clear dependence on latitudes, as shown in the inset of Fig. 3. In the same figure we also show the spectra calculated over lines of constant latitude. The power-law spectra indicate the existence of spatial patterns in the form of fractal surfaces. The two spectra in midlatitudes $\left(40^{\circ} \mathrm{N}\right.$ and $\left.40^{\circ} \mathrm{S}\right)$ exhibit a $1 / k^{2}$ spectrum as if they were $1 \mathrm{D} \mathrm{KPZ}$ surfaces. However, at the equator, $1 / k$ spectra are obtained, as found in a $2 \mathrm{D}$ EW model. A 


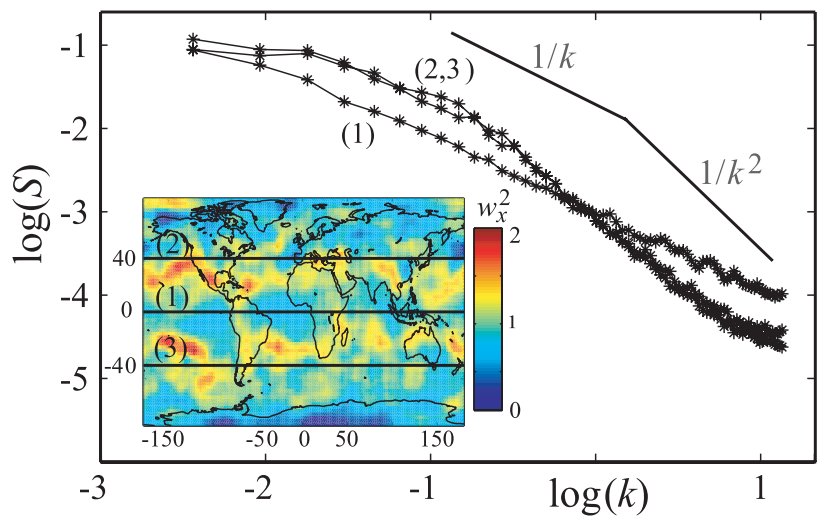

FIG. 3 (color online). Power spectra of logarithmic fluctuations calculated for the last forecast day of the medium-range forecast over constant latitude lines: Equator, $40^{\circ} \mathrm{N}$ and $40^{\circ} \mathrm{S}$. The inset shows the local width $w_{x}^{2}$ computed in $17.5^{\circ} \times 17.5^{\circ}$ windows centered at each point $x$.

naïve interpretation of these facts would suggest a $2 \mathrm{D}$ convective model with EW interfaces with two KPZ turbulent lines in the tropics. This agrees with what is expected from physical symmetries, but a more detailed analysis is necessary to confirm this hypothesis.

For comparison with the 1D coupled map lattice, Fig. 4 shows the total width $w^{2}$ and the logarithmic growth rate $\dot{E} / E$ against $\log E(t)$ for successive days $t$ of the EPS models. The trends of $w^{2}$ curves show clearly the two evolution stages, the initial infinitesimal growth with increasing width, where fluctuations become more correlated and localized, and the second stage where correlation decays due to saturation effects (the ensemble spread reaches the system amplitude). These curves are similar in form to those obtained in cases $A$ and $C$ for the 1D map lattice model. Note that $B$ corresponded to random spatially correlated fluctuations (e.g., bred vectors for a different condition) which do not correspond to the EPS models. Contrary to the $1 \mathrm{D}$ map lattice, the logarithmic growth rate is decreasing in all cases. This is due to anisotropy. An amount of correlation in these models implies an expansion of the zone with $1 / k^{2}$ spectrum over the convective one (with $1 / k$ spectrum), with the result of increasing the total width, but decreasing the contribution of the KPZ term, as shown in the inset to Fig. 4. The similar behaviors obtained for the logarithmic growth rate $\dot{E} / E$ and the KPZ term confirm the validity of (4) and the importance of the relative spatial fluctuations in the form of temporal patterns.

Summary. - We have presented a characterization of the spatiotemporal evolution of finite perturbations, based on well-known scaling techniques of rough surfaces. This framework allows us to analyze in more detail the curves observed in the growth rate, whose concave or convex shape has been previously associated with the existence of multiple scales in the system. We have shown that even

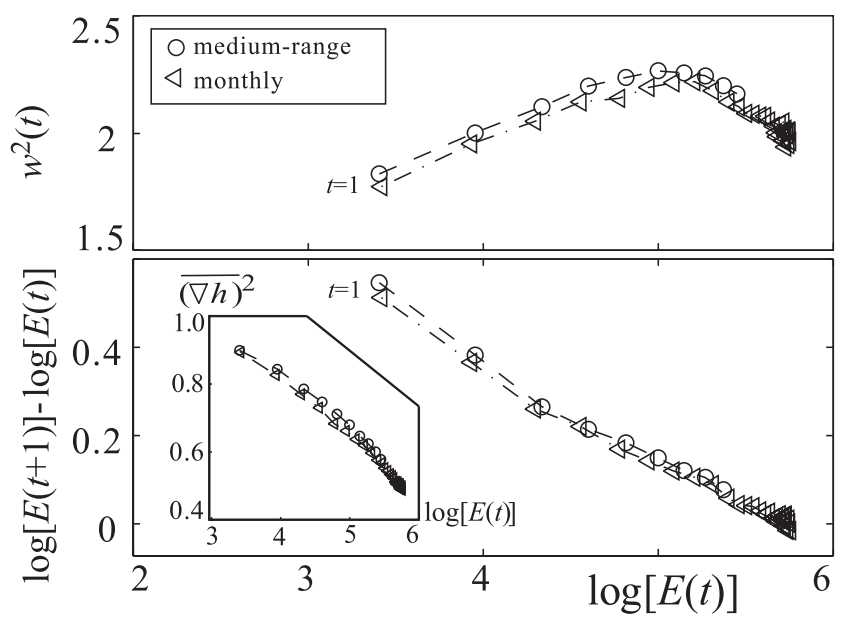

FIG. 4. Global width $w^{2}$ of the logarithmic fluctuations and the growth rate $\dot{E} / E$ computed for the two EPS models. The inset shows the KPZ term.

in simple models this shape depends on the choice of the initial perturbations, more specifically on their spatial correlation and localization, independently of the model's complexity. Future practical applications of these results are important for operational weather forecast to improve predictability by controlling the spatial patterns of initial perturbation, since they influence the temporal growth of the perturbation.

Financial support from the Ministerio de Ciencia y Tecnología (Spain) under Project No. CGL2004-02652/ CLI is acknowledged.

[1] T. Gneiting and A. E. Raftery, Science 310, 248 (2005).

[2] K. Kaneko, Physica (Amsterdam) 37D, 60 (1989).

[3] E. Lorenz, in Proceedings of a Seminar held at ECMWF on Predictability (European Centre for Medium-Range Weather Forecasts, Reading, UK, 1996), p. 118.

[4] J. Harlim, M. Oczkowski, J. A. Yorke, E. Kalnay, and B. R. Hunt, Phys. Rev. Lett. 94, 228501 (2005).

[5] A. Pikovsky and A. Politi, Nonlinearity 11, 1049 (1998).

[6] A norm is normally used to define the error amplitude. However, as we showed in [7], a more convenient definition of amplitude in multiplicative processes can be given considering the geometric mean, which is the norm in the logarithmic space.

[7] C. Primo, M. A. Rodríguez, J. M. López, and I. Szendro, Phys. Rev. E 72, 015201(R) (2005).

[8] A.L. Barabási and H.E. Stanley, Fractal Concepts in Surface Growth (Cambridge University Press, Cambridge, 1995).

[9] J. M. López, C. Primo, M. A. Rodríguez, and I. Szendro, Phys. Rev. E 70, 056224 (2004).

[10] J. Krug and P. Meaking, J. Phys. A 23, L987 (1990).

[11] R. Buizza, ECMWF Newsletter 104, 10 (2005); D. Anderson, T. Stockdale, L. Ferranti, and M. Balmaseda, ECMWF Newsletter 98, 17 (2003). 